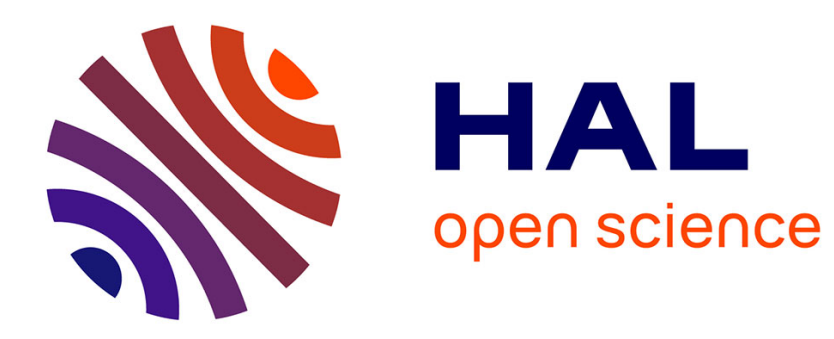

\title{
Minimum Size Tree-Decompositions
}

Bi Li, Fatima Zahra Moataz, Nicolas Nisse

\section{To cite this version:}

Bi Li, Fatima Zahra Moataz, Nicolas Nisse. Minimum Size Tree-Decompositions. 9th International colloquium on graph theory and combinatorics (ICGT), 2014, Grenoble, France. hal-01023904

\section{HAL Id: hal-01023904 \\ https://hal.inria.fr/hal-01023904}

Submitted on 15 Jul 2014

HAL is a multi-disciplinary open access archive for the deposit and dissemination of scientific research documents, whether they are published or not. The documents may come from teaching and research institutions in France or abroad, or from public or private research centers.
L'archive ouverte pluridisciplinaire HAL, est destinée au dépôt et à la diffusion de documents scientifiques de niveau recherche, publiés ou non, émanant des établissements d'enseignement et de recherche français ou étrangers, des laboratoires publics ou privés. 


\title{
Minimum Size Tree-Decompositions ${ }^{\star}$
}

\author{
Bi Li, Fatima Zahra Moataz, and Nicolas Nisse \\ 1 Inria, France \\ 2 Univ. Nice Sophia Antipolis, CNRS, I3S, UMR 7271, Sophia Antipolis, France
}

\begin{abstract}
Tree-Decompositions are the corner-stone of many dynamic programming algorithms for solving graph problems. Since the complexity of such algorithms generally depends exponentially on the width (size of the bags) of the decomposition, much work has been devoted to compute treedecompositions with small width. However, practical algorithms computing tree-decompositions only exist for graphs with treewidth less than 4. In such graphs, the time-complexity of dynamic programming algorithms based on tree-decompositions is dominated by the size (number of bags) of the treedecompositions. It is then interesting to try to minimize the size of the tree-decompositions.

In this extended abstract, we consider the problem of computing a tree-decomposition of a graph with width at most $k$ and minimum size. More precisely, we focus on the following problem: given a fixed $k \geq 1$, what is the complexity of computing a tree-decomposition of width at most $k$ with minimum size in the class of graphs with treewidth at most $k$ ? We prove that the problem is NP-complete for any fixed $k \geq 4$ and polynomial for $k \leq 2$. On going work also suggests it is polynomial for $k=3$.
\end{abstract}

\section{Introduction}

A tree-decomposition of a graph $G$ [11] is a way to represent $G$ by a family of subsets of its vertex-set organized in a tree-like manner and satisfying some connectivity property. The treewidth of $G$ measures the proximity of $G$ with a tree. More formally, a tree decomposition of $G=(V, E)$ is a pair $(T, \mathcal{X})$ where $\mathcal{X}=\left\{X_{t} \mid t \in V(T)\right\}$ is a family of subsets, called bags, of $V$, and $T$ is a tree, such that:

$-\bigcup_{t \in V(T)} X_{t}=V$;

- for any edge $u v \in E$, there is a bag $X_{t}$ (for some node $t \in V(T)$ ) containing both $u$ and $v$;

- for any vertex $v \in V$, the set $\left\{t \in V(T) \mid v \in X_{t}\right\}$ induces a subtree of $T$.

The width of a tree-decomposition $(T, \mathcal{X})$ is $\max _{t \in V(T)}\left|X_{t}\right|-1$ and its size is order $|V(T)|$ of $T$. The treewidth of $G$, denoted by $t w(G)$, is the minimum width over all possible tree-decompositions of $G$.

If $T$ is constrained to be a path, $(T, \mathcal{X})$ is called a path-decomposition of $G$. The pathwidth of $G$, denoted by $p w(G)$, is the minimum width over all possible path-decompositions of $G$.

Tree-Decompositions are the corner-stone of many dynamic programming algorithms for solving graph problems. As an example, the famous Courcelle's Theorem states that any problem expressible in MSOL can be solved in linear-time in the class of bounded treewidth graphs [5]. Another framework based on graph decompositions is the bi-dimensionality theory that allowed the design of sub-exponential-time algorithms for many problems in the class of graphs excluding some fixed graph as a minor (e.g., [6]). Given a tree-decomposition with width $w$ and size $n$, the time-complexity of most of such dynamic programming algorithms can be expressed as $O\left(2^{w} n\right)$ (or $O\left(2^{w \log w} n\right)$ in the case of global problems). Therefore, the problem of computing tree-decompositions with small width has drawn much attention in the last decades. It has been extensively studied and investigated from different angles: parametrized complexity, exact or approximation algorithms.

The above mentioned algorithms have mainly a theoretical interest because, on the one hand, their timecomplexity exponential depends on the treewidth of graphs and, on the other hand, as far as we know, no practical algorithm exists that computes a "good" tree-decomposition for graphs with treewidth at least 5 . However, in case of small $(\leq 4)$ treewidth graphs, efficient (i.e., practical) algorithms exist to compute 
tree-decompositions with optimal width. Moreover, in such case, the time-complexity of above-mentioned dynamic programming algorithms becomes dominated by the size of the tree-decompositions and, therefore, it becomes interesting to minimize it.

In this extended abstract, we deal with the problem of computing tree-decompositions with minimum size. Obviously, if the width is not constrained, then the problem is trivial since there always exists a treedecomposition of a graph with one bag (the full vertex-set). Hence, given a graph $G$ and an integer $k \geq t w(G)$, we consider the problem of minimizing the size of a tree-decomposition of $G$ with width at most $k$.

Our results. Let $k$ be any positive integer and $G$ be any graph with treewidth at most $k$. Let $s_{k}(G)$ denote the smallest size of a tree-decomposition of $G$ with width at most $k$. We first prove that, for any (fixed) $k \geq 4$, the problem of computing $s_{k}$ is NP-hard in the class of graphs with treewidth at most $k$. Then, we prove that computing $s_{2}$ can be solved in polynomial-time in the class of graphs with treewidth at most 2 .

Related Work. The problem of computing "good" tree-decompositions has been extensively studied. Computing optimal tree-decomposition - i.e., with width $t w(G)$ - is NP-complete in the class of general graphs $G$ [1]. For any fixed $k \geq 1$, Bodlaender designed an algorithm that computes, in time $O\left(k^{k^{3}} n\right)$, a treedecomposition of width $k$ of any $n$-node graph with treewidth at most $k$ [3]. Very recently, a single-exponential (in $k$ ) algorithm has been proposed that computes a tree-decomposition with width at most $5 k$ in the class of graphs with treewidth at most $k$ [4]. As far as we know, the only practical algorithms for computing optimal tree-decompositions hold for graphs with treewidth at most 1 (trivial since $t w(G)=1$ if and only if $G$ is a tree), 2 (graphs excluding $K_{4}$ as a minor) [13], 3 [2,9,10] and 4 [12].

We are not aware of any work dealing with the computation of tree-decompositions with minimum size. In [7], Dereniowski et al. consider the problem of size-constrained path-decompositions. Given any positive integer $k$ and any graph $G$ with pathwidth at most $k$. Let $l_{k}(G)$ denote the smallest size (length) of a pathdecomposition of $G$ with width at most $k$. For any fixed $k \geq 4$, computing $l_{k}$ is NP-complete in the class of general graphs and it is NP-complete, for any fixed $k \geq 5$, in the class of connected graphs [7]. Moreover, computing $l_{k}$ can be solved in polynomial-time in the class of graphs with pathwidth at most $k$ for any $k \leq 3$. Finally, the "dual" problem is also hard: for any fixed $s \geq 2$, it is NP-complete in general graphs to compute the minimum width of a tree-decomposition with size $s[7]^{3}$.

\section{NP-hardness in the class of graphs with treewidth at least 4}

In this section, we prove that:

Theorem 1. For any fixed integer $k \geq 4$ (resp., $k \geq 5$ ), the problem of computing $s_{k}$ is NP-complete in the class of graphs (resp., of connected graphs) with treewidth at most $k$.

Note that the corresponding decision problem is clearly in NP, hence, we only need to prove it is NP-hard.

Our proof mainly follows the one of [7] for size-constrained path-decompositions. Hence, we recall here the two steps of the proof in [7]. First, it is proved that, if computing $l_{k}$ is NP-hard for some $k \geq 4$ in general graphs, then the computation of $l_{k+1}$ is NP-hard in the class of connected graphs. Then, it is shown that computing $l_{4}$ is NP-hard in general graphs with pathwidth at least 4 , resp., computing $l_{5}$ is NP-hard in the class of connected graphs with pathwidth at least 5 . The second step consists of a reduction from the 3 -PARTITION problem [] to the one of computing $l_{4}$ (resp., of $l_{5}$ in connected graphs). Precisely, for any instance $\mathcal{I}$ of 3 -PARTITION, a graph $G_{\mathcal{I}}$ is built such that $\mathcal{I}$ is a YES instance if and only if $l_{4}\left(G_{\mathcal{I}}\right)$ equals some defined value $\ell_{\mathcal{I}}$.

Our contribution consists first in showing that the first step of [7] directly extends to the case of treedecompositions. That is, it directly implies that, if computing $s_{k}$ is NP-hard for some $k \geq 4$ in general graphs, then the computation of $s_{k+1}$ is NP-hard in connected graphs. Our main contribution of this section is to show that, for the graphs $G_{\mathcal{I}}$ built in the reduction proposed in [7], any tree-decomposition of $G_{\mathcal{I}}$ with width at most 4 and minimum size is a path decomposition. Hence, in this class of graphs, $l_{4}=s_{4}$ and, therefore, for any instance $\mathcal{I}$ of 3-PARTITION, $\mathcal{I}$ is a YES instance if and only if $s_{4}\left(G_{\mathcal{I}}\right)$ equals $\ell_{\mathcal{I}}$. Theorem 1 follows.

\footnotetext{
${ }^{3}$ This result was proved in [7] in terms of path-decomposition but it is straightforward to extend it to treedecomposition.
} 


\section{Polynomial cases}

In this section, we give preliminary results on when minimum size tree-decompositions can be computed in polynomial-time. We first investigate the case of forests.

Theorem 2. For any $k \in\{1,2,3\}$, a tree-decomposition with size $s_{k}(F)$ and width $k$ can be computed in polynomial-time in the class of forests $F$.

Note that the computation of $s_{1}$ is trivially polynomial since, in particular, $s_{1}(T)=n-1$ for any $n$-node tree. For $k \in\{2,3\}$, we design polynomial-time algorithms for computing $s_{k}$ in the class of forests. These are recursive algorithms that proceed greedily. Intuitively, in any forest $F$, we can identify a subgraph $S$ of size at most $k+1$ such that $S$ is a bag in a minimum-size tree-decomposition of $F$ with width $k$.

Due to lack of space, we only give an example in the case $k=3$. Let $F$ be a forest and let $v \in V(F)$ adjacent to exactly one non-leaf node. Moreover, assume that $v$ is adjacent to at least three leaves $a, b, c \in V(F)$. Our algorithm first computes (recursively) a minimum-size tree-decomposition $\mathcal{T}$ of $F \backslash\{a, b, c\}$ with width at most 3. Let $B$ be any bag of $\mathcal{T}$ containing $v$. We prove that the tree-decomposition obtained from $\mathcal{T}$ by adding a bag $\{v, a, b, c\}$ adjacent to $B$ is a minimum-size tree-decomposition of $F$ with width 3 .

The key point is that the number of cases to consider is relatively small. In particular, in the case of trees, we prove that there always exists a minimum-size tree-decomposition with width at most $k \leq 3$ where each bag induces a (connected) subtree. That is, in case of trees, the cases to consider are all trees with at most 4 nodes. It seems that our algorithms cannot be easily extended for $k \geq 4$ since, in particular, this connectivity property is not valid anymore for $k>4$ (see conclusion).

Then, we focus on graphs with treewidth 2.

Theorem 3. A tree-decomposition of size $s_{2}(G)$ and width 2 can be computed in polynomial-time in the class of graphs $G$ with treewidth 2.

The first step of the proof of the above theorem is to consider 2-connected graphs with treewidth 2 . It is known that any 2-connected graph has treewidth 2 if and only if it has an open nested ear decomposition starting from a single edge [8]. In particular, this implies that any such a graph contains a node with degree 2. Given a 2-connected graph $G$ with treewidth 2, let $v$ be a node with degree 2 and $u$ and $w$ its neighbors. Let $G^{\prime}$ obtained from $G$ by contracting the edge $\{u, v\}$ (or equivalently, removing $v$ and adding an edge between $u$ and $w$ ). Our algorithm first computes (recursively) a minimum-size tree-decomposition $\mathcal{T}$ of $G^{\prime}$ with width 2 . One bag $B$ of it contains $\{u, w\}$. We prove that the tree-decomposition obtained from $\mathcal{T}$ by adding a bag $\{v, u, w\}$ adjacent to $B$ is a minimum-size tree-decomposition of $G$ with width 2 .

Then, we consider the case of general graphs with treewidth 2. Given such a graph $G$, let $G_{1}, \cdots, G_{r}$ be its blocks, i.e., its inclusion-maximal 2-connected components and let $G^{\prime}$ be the graph obtained by removing all edges of the $G_{i} \mathrm{~s}(i \leq r)$ and removing all nodes that are not cut-vertices. By definition $G^{\prime}$ is a forest. We prove that a minimum-size tree-decomposition of $G$ with width 2 can easily be obtained by combining minimum-size tree-decompositions with width 2 of the subgraphs $G_{i}$ (computable in polynomial-time by above paragraph) and of $F$ (using Theorem 2). More precisely, we prove that there always exists a minimumsize tree-decomposition of $G$ with width 2 that does not contain mixed bags, i.e., bags containing two nodes in some $G_{i}$ and one node not in $G_{i}$.

Finally, we consider the case $k=3$ for graphs with treewidth 2 . We prove that:

Theorem 4. A tree-decomposition of size $s_{3}(G)$ and width at most 3 can be computed in polynomial-time in the class of 2-connected graphs $G$ with treewidth 2 .

Unfortunately, in the case $k=3$, minimum-size tree-decomposition with width 3 may always contain mixed bags. This makes the computation of $s_{3}$ in the case of connected graphs with treewidth 2 more tricky. We are currently investigated this case. 


\section{Conclusion}

In this extended abstract, we gave preliminary results on the complexity of minimizing the size of treedecompositions with given width. Table 1 summarizes our results as well as the remaining open questions. We currently investigate the case of $s_{3}$ in the class of graphs with treewidth 2 or 3 and we conjecture it is polynomially solvable. The problem of computing $s_{k}$, for $k \geq 4$, seems more intricate already in the case of trees. Indeed, our polynomial-time algorithms to compute $s_{k}, k \leq 3$, in trees mainly rely on the fact that, for any tree $T$, there exists a minimum-size tree-decomposition of $T$ with width at most 3 , where each bag induces a connected subtree. This is unfortunately not true anymore in the case of tree-decomposition with width 5 . As an example, consider the tree (with 10 nodes) obtained from a star with three 3 leaves by subdividing twice each edge.

\begin{tabular}{|l|c|c|c|c|}
\cline { 2 - 5 } \multicolumn{1}{c|}{} & $s_{1}$ & $s_{2}$ & $s_{3}$ & $s_{k}, k \geq 4$ \\
\hline$t w=1$ & $P($ trivial $)$ & $P$ & $P$ & $?$ \\
\hline$t w=2$ & - & $P$ & $?$ & $?$ \\
\hline$t w=3$ & - & - & $?$ & $?$ \\
\hline$t w \geq 4$ & - & - & - & NP-hard \\
\hline
\end{tabular}

Table 1. Summary of the complexity results.

\section{References}

1. Arnborg, S., Corneil, D. G., And Proskurowski, A. Complexity of finding embeddings in a k-tree. SIAM J. Algebraic Discrete Methods 8, 2 (Apr. 1987), 277-284.

2. Arnborg, S., And Proskurowski, A. Characterization and recognition of partial 3-trees. SIAM J. Algebraic Discrete Methods 7, 2 (Apr. 1986), 305-314.

3. Bodlaender, H. L. A linear-time algorithm for finding tree-decompositions of small treewidth. SIAM J. Comput. 25, 6 (1996), 1305-1317.

4. Bodlaender, H. L., Drange, P. G., Dregi, M. S., Fomin, F. V., Lokshtanov, D., And Pilipczuk, M. A $o\left(c^{k} n\right)$ 5-approximation algorithm for treewidth. CoRR abs/1304.6321 (2013).

5. Courcelle, B. The monadic second-order logic of graphs. i. recognizable sets of finite graphs. Information and Computation 85, 1 (1990), $12-75$.

6. Demaine, E. D., And Hajiaghayi, M. The bidimensionality theory and its algorithmic applications. Comput. J. 51, 3 (2008), 292-302.

7. Dereniowski, D., Kubiak, W., And Zwols, Y. Minimum length path decompositions. CoRR abs/1302.2788 (2013).

8. Eppstein, D. Parallel recognition of series-parallel graphs. Inf. Comput. 98, 1 (May 1992), 41-55.

9. Kajitani, Y., Ishizuka, A., And Ueno, S. Characterization of partial 3-trees in terms of three structures. Graphs and Combinatorics 2, 1 (1986), 233-246.

10. Matousek, J., And Thomas, R. Algorithms finding tree-decompositions of graphs. Journal of Algorithms 12, 1 (1991), $1-22$.

11. Robertson, N., And Seymour, P. D. Graph minors. ii. algorithmic aspects of tree-width. J. Algorithms 7, 3 (1986), 309-322.

12. Sanders, D. P. On linear recognition of tree-width at most four. SIAM J. Discret. Math. 9, 1 (1996), $101-117$.

13. Wald, J. A., And Colbourn, C. J. Steiner trees, partial 2-trees, and minimum ifi networks. Networks 13,2 (1983), 159-167. 